\title{
Life in slow motion: quantifying physical activity in COPD
}

\section{Mike Morgan}

We probably all acknowledge that regular spontaneous physical activity plays a large role in maintaining health and physical fitness. In health, the promotion of regular physical activity has been a feature of active living since at least the time of Ancient Greece. In more modern times we have continued this tradition by encouraging our children to play sport and sustaining recreational physical activity through to old age.

Although the health benefits of maintaining spontaneous physical activity in otherwise healthy people are evident, it is only in more recent times that we have realised its importance in people with chronic disease. In people with chronic obstructive pulmonary disease (COPD), the reduction of physical activity is both a consequence and a symptom. Advancing airway obstruction leads to breathlessness that impedes activities of daily living and may naturally be compounded by the physical deconditioning induced by inactivity. We now understand that reduced physical activity is associated with a poorer prognosis in those with COPD, as well as a higher risk of hospital admission. ${ }^{1-3}$ In addition, therapies such as pulmonary rehabilitation are beneficial because they improve health status indirectly through the medium of physical exercise training and the increase in functional exercise capacity. Apart from slowing down the rate of decline of airway function, the main aims of treatment in COPD are to increase the quality of life and to improve symptoms of breathlessness during activity. To have a sustained beneficial effect, any therapy will have to bequeath a change in lifestyle which results in increased spontaneous physical activity. The simple provision of improvements in airway function or physical exercise capacity is insufficient to ensure sustained benefit unless the new capacity continues to be used. The recording of spontaneous daily physical activity therefore adds a different dimension to patient assessment that goes beyond any

Correspondence to: Dr M D L Morgan, Department of Respiratory Medicine, University Hospitals of Leicester NHS Trust, Glenfield Hospital, Leicester LE3 9QP; mike.morgan@uhl-tr.nhs.uk measurement of physiological capacity. The distinct concepts of physiological capacity and the volitional or habitual use of that capacity were described some years ago by Leidy. ${ }^{4}$ To this end, we have to consider what people actually do rather than what they are capable of doing, since it is the natural level of physical activity that seems to determine the prognostic benefit. We therefore have to develop objective methods of quantifying daily activity that can be used to record therapeutic outcome.

The assessment of daily physical activity first requires a notion of what is understood by the term. The World Heath Organization defines physical activity as "any bodily movement produced by the skeletal muscles that results in energy expenditure". This will obviously vary significantly depending upon the context. The spectrum of daily physical activity may include competitive or recreational sporting activity, occupational duties or necessary domestic tasks such as cleaning and shopping. For patients with COPD, the challenge may simply be to conduct personal hygiene tasks and achieve basic mobility around the home. The quantification of daily physical activity therefore presents a challenge, particularly at the sedentary end of the patient spectrum where even small changes in physical activity may have significant gains for health status and independence. The methods that are available to quantify physical activity in daily life include direct observation, assessment of energy expenditure and the use of physical activity questionnaires and motion sensors. Direct observation and detailed measurement of energy expenditure are not really practical tools for research trials or clinical practice but questionnaires and motion sensors have more promise. This has recently been the subject of an excellent review by Pitta et al that describes the qualities of questionnaires and motion sensors. ${ }^{5}$ Questionnaire or diary methods of assessing physical activities are cheap and easily applicable. Although some questionnaires have been used in people with COPD, there are some intrinsic difficulties that make them less attractive than direct measurement of physical activity. The questionnaires depend upon recall that may be insecure, particularly when the activities concerned are low or very low intensity as will be the case in patients with COPD. The ability to detect change will also be limited. For these reasons the use of physical activity questionnaires may be better confined to epidemiological surveys. For individual assessments the motion sensors hold much greater promise as a potential outcome assessment.

The science of motion detection in relation to COPD is in its relative infancy, but some principles are beginning to emerge. The devices that are currently available can measure average activity over a period, intensity and timing of activities and, in some cases, the type of physical activity performed. Some of these can also use additional sensors to estimate energy expenditure. These features enable us to describe the patterns of activity in subjects with COPD and compare them with healthy subjects. It also allows the observation of the effects of therapeutic interventions. The available devices vary in complexity and cost. There are simple pedometers, accelerometers, devices capable of deriving energy expenditure and more sophisticated activity monitors that can distinguish different types of activity. Now that there has been some experience with these devices, it is time to take stock and consider what can be learnt from motion sensors and perhaps what level of technology may be appropriate.

The pedometer is a simple mechanical device that records movement in a vertical direction. The invention is credited to various people including Thomas Jefferson and Robert Hooke, circa 1674. It is essentially a step counter and has received popular attention as an aid to maintaining a healthy level of activity, as in the ten thousand steps campaign. These devices have been shown to have some reproducibility in people with COPD but may not be as sensitive to movement in patients as normal subjects. $^{67}$ It is likely that the vertical acceleration required by the pedometer will not always be achieved by the lower intensity of movement in the disabled patient. Accelerometers are more sensitive devices that can measure acceleration in the single vertical plane (uniaxial) or in additional two or three planes (bi- and triaxial). The devices can generally store the data and present the output as periodic intensity in addition to total activity. 
These devices have significant advantages over the pedometer but also have some drawbacks. The accelerometers may pick up different information depending upon where they are placed on the body. They may also pick up signals from extraneous movements such as car vibration. Apart from the additional cost, the monitors may have individual characteristics that require the same monitor to be used for repeat measurements. The current peak of sophistication with motion sensors is the Dynaport activity monitor that has a leg position sensor in addition to the waist accelerometer. This can characterise the type of activity and body position in addition to the overall intensity of motion, and has been shown to be as accurate as direct observation. ${ }^{8}$

There is now a reasonable amount of experience with motion sensors in COPD and this has begun to produce interesting data. For example, we now know just how inactive people with COPD can become, particularly if they are using long-term oxygen therapy.9 The pattern of daily activity in COPD shows that patients spend proportionately more time sitting and lying. ${ }^{10}$ Improvements in the quantity of daily activity following pulmonary rehabilitation have also been described, as well as the monitoring of compliance with exercise training. ${ }^{11} 12$ For the future, the areas to explore might include seasonal or gender variation in activity as well as the effects of other therapies such as drugs or ambulatory oxygen.

The paper published in this issue of Thorax by Walker et al ${ }^{13}$ further enhances our experience of the technology (see page 683). The authors have explored the relationship in COPD between lower limb activity and total daily activity as well as the relationship with more routine assessments. In the first of three evaluations the authors find that lower limb activity measured by a uniaxial accelerometer over 2 days is an accurate reflection of whole body activity which is described by the more comprehensive Dynaport activity monitor. Interestingly, this observation confirms other evidence that shows that the lower limb musculature is the major victim of muscular deconditioning in COPD. ${ }^{14}$ It also suggests that rehabilitation strategies should focus on this area. The authors then argue that the uniaxial accelerometer on the lower limb is sufficient for the remaining evaluations. A comparison with healthy subjects confirms that patients spend approximately $10 \%$ less time mobile, with $40 \%$ less activity and lower intensity. After a comprehensive rehabilitation programme that included lower limb strength and endurance exercise, there was a marked improvement in all three elements of daily activity as well as the usual clinically significant improvements in quality of life and 6 min walk distance. What was surprising was that there appeared to be no relationship between the improvements in functional capacity (6 min walk test) or health status (St George's Questionnaire) and the changes in spontaneous daily activity. There was, however, an apparent relationship between airway function (forced expiratory volume in $1 \mathrm{~s}, \mathrm{FEV}_{1}$ ) and the improvement in daily activity. From the data it appears as if this correlation is much tighter at lower levels of $\mathrm{FEV}_{1}$, but much less so when airway function is better. This is not really too surprising since physical activity may be predominantly constrained by dynamic hyperinflation at this level of lung function and not improve that much, so it is important to know whether breathlessness during daily tasks is modified by rehabilitation. Interestingly, this area has also recently been explored by a German research group who examined, in a cross-sectional study, the relationship between daily physical activity and numerous pulmonary and extrapulmonary factors. ${ }^{15}$ They concluded that extrapulmonary factors - in particular, systemic inflammation and cardiac disease-made a significant contribution to physical inactivity. However, there is no contradiction in this observation since the patients in this study had better lung function and were presumably less constrained by their poor ventilatory limitation.

So what can we infer from all this? The first thing is that it does appear to be possible to quantify daily physical activity objectively in people with COPD who have developed low levels of activity. It also does not seem necessary to use overly complicated devices if lower limb activity is the key determinant of whole body activity. Poor airway function may be responsible for low levels of physical activity when the $\mathrm{FEV}_{1}$ is low, but other physiological or behavioural factors may influence the level of spontaneous activity in less impaired patients. However, spontaneous daily activity is clearly a different domain of outcome assessment from the traditional measures of airway function, functional exercise capacity and quality of life. As we become more confident with these activity measurements, we should be able to develop a greater insight into the lives of people who are disabled by breathlessness and offer treatment that really gets to the heart of the matter.

Competing interests: None.

Thorax 2008;63:663-664. doi:10.1136/thx.2007.093492

\section{REFERENCES}

1. Garcia-Aymerich J, Lange $P$, Benet $M$, et al. Regular physical activity modifies smoking-related lung function decline and reduces risk of chronic obstructive pulmonary disease: a population-based cohort study. Am J Respir Crit Care Med 2007;175:458-63.

2. Garcia-Aymerich J, Lange $P$, Benet $M$, et al. Regular physical activity reduces hospital admission and mortality in chronic obstructive pulmonary disease: a population based cohort study. Thorax 2006;61:772-8.

3. Pitta F, Troosters T, Probst VS, et al. Physical activity and hospitalization for exacerbation of COPD. Chest 2006;129:536-44.

4. Leidy NK. Using functional status to assess treatment outcomes. Chest 1994;106:1645-6.

5. Pitta F, Troosters T, Probst VS, et al. Quantifying physical activity in daily life with questionnaires and motion sensors in COPD. Eur Respir J 2006;27:1040-55.

6. Schonhofer B, Ardes P, Geibel M, et al. Evaluation of a movement detector to measure daily activity in patients with chronic lung disease. Eur Respir J 1997;10:2814-9.

7. Tudor-Locke C, Ainsworth BE, Thompson RW, et al. Comparison of pedometer and accelerometer measures of free-living physical activity. Med Sci Sports Exerc 2002;34:2045-51

8. Pitta F, Troosters T, Spruit MA, et al. Activity monitoring for assessment of physical activities in daily life in patients with chronic obstructive pulmonary disease. Arch Phys Med Rehabil 2005;86:1979-85.

9. Sandland CJ, Singh SJ, Curcio A, et al. A profile of daily activity in chronic obstructive pulmonary disease. J Cardiopulm Rehabil 2005;25:181-3.

10. Pitta F, Troosters T, Spruit MA, et al. Characteristics of physical activities in daily life in chronic obstructive pulmonary disease. Am J Respir Crit Care Med 2005;171:972-7.

11. Sewell L, Singh SJ, Williams JE, et al. Can individualized rehabilitation improve functional independence in elderly patients with COPD? Chest 2005; 128:1194-200.

12. Hunter J, Singh SJ, Morgan MDL. Objective monitoring of adherence with home exercise training during pulmonary rehabilitation for COPD.

Physiotherapy 2006;92:50-4.

13. Walker PP, Burnett A, Flavahan PW, et al. Lower limb activity and its determinants in COPD. Thorax 2008;63:683-9.

14. Swallow EB, Reyes D, Hopkinson NS, et al. Quadriceps strength predicts mortality in patients with moderate to severe chronic obstructive pulmonary disease. Thorax 2007;62:115-20.

15. Watz H, Waschki B, Boehme C, et al. Extrapulmonary effects of chronic obstructive pulmonary disease on physical activity: a cross-sectional study. Am J Respir Crit Care Med 2008;177:743-51. 